Bull. Austral. Math. Soc.

$16 \mathrm{~s} 30,17 \mathrm{~B} 50,17 \mathrm{~B} 56$

Vol. 75 (2007) [27-44]

\title{
ON POWERFUL AND p-CENTRAL RESTRICTED LIE ALGEBRAS
}

\section{S. Siciliano AND Th. Weigel}

In this note we analyse the analogy between $m$-potent and $p$-central restricted Lie algebras and $p$-groups. For restricted Lie algebras the notion of $m$-potency has stronger implications than for $p$-groups (Theorem A). Every finite-dimensional restricted Lie algebra $\mathfrak{L}$ is isomorphic to $\tilde{\mathfrak{L}} / \widetilde{\mathfrak{L}}_{[p]}$ for some finite-dimensional p-central restricted Lie algebra $\tilde{\mathfrak{L}}$ (Proposition $\mathrm{B}$ ). In particular, for restricted Lie algebras there does not hold an analogue of J.Buckley's theorem. For $p$ odd one can characterise powerful restricted Lie algebras in terms of the cup product map in the same way as for finite $p$-groups (Theorem C). Moreover, the $p$-centrality of the finite-dimensional restricted Lie algebra $\mathfrak{L}$ has a strong implication on the structure of the cohomology ring $H^{\bullet}(\mathfrak{L}, \mathbb{F})$ (Theorem D).

\section{INTRODUCTION}

The structure theory of powerful $p$-groups had a strong impact on the study of finite and infinite pro- $p$ groups (see $[15,16]$ ). Moreover, the mod $p$ cohomology of $p$-central groups has been studied quite intensively, since for these groups the cohomology ring $H^{\bullet}\left(G, \mathbb{F}_{p}\right)$ is easiest to analyse (see $\left.[6,28]\right)$. In this note we shall analyse these concepts for restricted Lie algebras.

One would expect that powerful restricted Lie algebras play a similar role in the category of finite-dimensional p-nilpotent restricted Lie algebras as powerful $p$-groups play in the category of finite $p$-groups. However, this is not the case. Let $F$ be a field of characteristic $p>0$, and let $\mathfrak{F}_{p}$ denote the class of finite-dimensional $p$-nilpotent restricted $\mathbb{F}$-Lie algebras. For $p \neq 2$, the restricted Lie algebra $\mathfrak{L} \in \mathfrak{F}_{p}$ is called $m$-potent, $m<p-1$, if

$$
\gamma_{m+1}(\mathfrak{L}) \leqslant \mathfrak{L}^{[p]}
$$

where $\gamma_{k}(\mathfrak{L})$ denotes the $k^{\text {th }}$-term of the descending central series of $\mathfrak{L}$, and $\mathfrak{L}^{[p]^{i}}$ denotes the $\mathbb{F}$-vector space spanned by the elements $x^{[p]^{i}}, x \in \mathfrak{L}$. So 1-potent restricted Lie algebras are just powerful restricted Lie algebras as introduced by Riley and Semple in Received 4th July, 2006

Copyright Clearance Centre, Inc. Serial-fee code: 0004-9727/07 \$A2.00+0.00. 
[19]. For $m=p-2$, our definition is in analogy with the definition used by GonzálezSánchez and Jaikin-Zapirain for $p$-groups (see [9]). For $p=2, \mathfrak{L}$ is called 1-potent-or powerful - if

$$
[\mathfrak{L}, \mathfrak{L}] \leqslant\left(\mathfrak{L}^{[2]}\right)^{[2]} .
$$

Obviously, there exist powerful $p$-groups of arbitrary high nilpotency class. However, for restricted Lie algebras one has the following (see.Theorem 2.6, Proposition 2.7).

THEOREM A. (a) Let $\mathfrak{L} \in \mathfrak{F}_{p}$ be an $m$-potent restricted Lie algebra, $m<p-1$ for $p$ odd, or $m=1$ for $p=2$. Then $\mathfrak{L}$ is nilpotent of class $\operatorname{cl}(\mathfrak{L}) \leqslant m+1$. Moreover, $\mathfrak{L}^{[p]^{3}}$ is a restricted Lie ideal for all $j \geqslant 0$, and one has $\left(\mathfrak{L}^{\left[p^{i}\right]}\right)^{[p]^{j}}=\mathfrak{L}^{[p]^{i+j}}$. In particular, if $\mathbb{F}$ is perfect, then for every $x \in \mathfrak{L}^{[p]^{j}}$ there exists $y \in \mathfrak{L}$ such that $x=y^{[p]^{j}}$.

(b) Let $p \neq 2$, let $\mathfrak{L}$ be a finite-dimensional $p$-nilpotent restricted Lie algebra, and let $d:=d(\mathfrak{L})$ denote the minimal number of generators of $\mathfrak{L}$ as restricted Lie algebra. Then $\mathfrak{L}$ is powerful, if and only if $\mathfrak{L}$ is a sum of $d$ cyclic restricted Lie algebras.

For $m=1$, the first part of Theorem A(a) has been proved already in [19, Section 5]. In section 3 we shall apply Theorem $A$ in order to analyse properties of the restricted universal enveloping algebras of these algebras.

While $m$-potency has much stronger implications for restricted Lie algebras than for p-groups, the implications for $p$-centrality are sometimes stronger and sometimes weaker. A restricted Lie algebra $\mathfrak{L}$ is called $p$-central, if

$$
\mathfrak{L}_{[p]}:=\left\{x \in \mathfrak{L} \mid x^{[p]}=0\right\} \leqslant Z(\mathfrak{L}),
$$

where $Z(\mathfrak{L})$ denotes the centre of the restricted Lie algebra $\mathfrak{L}$. Hence, for a $p$-central restricted Lie algebra $\mathfrak{L}$, the subset $\mathfrak{L}_{[p]}$ is a restricted Lie ideal in $\mathfrak{L}$. Finite-dimensional restricted Lie algebras have the following property (see Proposition 2.10).

PROPOSITION B. Let $\mathfrak{L}$ be a restricted Lie algebra of dimension $n<\infty$. Then there exists a p-central restricted Lie algebra $\tilde{\mathfrak{L}}$ of dimension $2 n$ such that $\mathfrak{L} \simeq \widetilde{\mathfrak{L}} / \tilde{\mathfrak{L}}_{[p]}$.

This property of restricted Lie algebras is in contrast to the situation one has for finite groups. Indeed, for $p$ odd, Buckley's theorem states that for a finite $p$-central group $G$ the group $G / \Omega_{1}(G)$ is $p$-central as well (see [7]). This phenomenon is also reflected by the fact that the characterisation of $p$-centrality given by Bianchi, Gillo Berta Mauri and Verardi (see [5]) for $p$-groups does not hold for restricted Lie algebras (see Proposition 2.11).

In the last section of the paper we consider cohomological properties of powerful and $p$-central restricted Lie algebras. For $p$ odd, one can characterise powerful restricted Lie algebras in the class $\mathfrak{F}_{p}$ (see Theorem 4.1) in the same way as one can characterise finitely generated powerful pro- $p$ groups in the class of all finitely generated pro- $p$ groups (see [27, Theorem 5.1.6]).

ThEOREM C. Let $p$ be odd and let $\mathfrak{L} \in \mathfrak{F}_{p}$. Then the following are equivalent: 
(i) $\mathfrak{L}$ is powerful.

(ii) Cup product induces an injective map

$$
-\cup_{-}: H^{1}(\mathfrak{L}, \mathbb{F}) \wedge H^{1}(\mathfrak{L}, \mathbb{F}) \rightarrow H^{2}(\mathfrak{L}, \mathbb{F}),
$$

where $\mathbb{F}$ denotes the trivial left $\mathfrak{u}(\mathfrak{L})$-module.

In [6], Broto and Henn showed that for an arbitrary $p$-central finite group $G$ the cohomology ring $H^{\bullet}\left(G, \mathbb{F}_{p}\right)$ is a Cohen-Macaulay $\mathbb{F}_{p}$-algebra. Let $p$ be odd. A finite group $G$ satisfies the $\Omega$-extension property, if there exists a finite p-central group $\widetilde{G}$ such that $G$ is isomorphic to $\widetilde{G} / \Omega_{1}(\widetilde{G})$. In [28, Theorem A] it was shown that a finite p-group $G$ satisfies the $\Omega$-extension property, if and only if

$$
H^{\bullet}\left(G, \mathbb{F}_{p}\right) \simeq C^{\bullet} \otimes_{\mathbf{F}_{p}} S^{\bullet}
$$

where $C^{\bullet}$ is a finite-dimensional graded commutative $\mathbb{F}_{p}$-algebra, and $S^{\bullet}$ is a polynomial algebra generated in degree 2. Another interpretation of Proposition B is that for restricted Lie algebras the $\Omega$-extension property is always satisfied. For restricted Lie algebras, we shall prove the following theorem (see Theorem 4.3, Corollary 4.5) which can be seen as an analogue of $[\mathbf{2 8}$, Theorem A].

TheOREm $\mathrm{D}$. Let $p$ be odd and let $\mathfrak{L}$ be a finite-dimensional restricted $p$-central restricted Lie algebra. Then

$$
H^{\bullet}(\mathfrak{L}, \mathbb{F}) \simeq C^{\bullet} \otimes_{\mathbf{F}} S^{\bullet}\left(\mathcal{L}_{[p]}^{*}\right)
$$

where $S^{\bullet}\left(\mathfrak{L}_{[p]}^{*}\right)$ is the polynomial $\mathbb{F}$-algebra generated by $\mathfrak{L}_{[p]}^{*}:=\operatorname{Hom}_{\mathbf{F}}\left(\mathfrak{L}_{[p]}, \mathbb{F}\right)$ in degree 2 , and $C^{*}$ is a finite-dimensional $\mathbb{F}$-algebra satisfying Poincare duality in dimension $n:=\operatorname{dim}_{\mathbb{F}_{p}}\left(\mathfrak{L}_{[p]}\right)$. In particular, $H^{\bullet}(\mathfrak{L}, \mathbb{F})$ is a Cohen-Macaulay $\mathbb{F}$-algebra.

If $p$ is odd, one can characterise finite $p$-groups with the $\Omega$-extension property by the structure of their cohomology ring (see [28]). Therefore, one would like to know whether the following problem has an affirmative answer.

Problem 1. Let $p$ be odd and let $\mathfrak{L} \in \mathfrak{F}_{p}$. Assume that $H^{\bullet}(\mathfrak{L}, \mathbb{F}) \simeq C^{\bullet} \otimes S^{\bullet}$, where $C^{\bullet}$ is a finite-dimensional $\mathbb{F}$-algebra, and $S^{\bullet}$ is a polynomial $\mathbb{F}$-algebra generated in degree 2 . Is it true that $\mathfrak{L}$ is $p$-central?

The main purpose of this paper is the study of $m$-potent restricted Lie algebras and $p$-central restricted Lie algebras in analogy to $m$-potent $p$-groups and $p$-central finite groups. However, there might be other contexts where these notions play an important role. We close the introduction with the following two open problems [The authors thank the referee for communicating these problems to them.] which might be the subject of further investigations.

Problem 2. Investigate $m$-potent and $p$-central restricted Lie algebras represented as ring constructions defined in [14, Chapter 3$]$. 
PROBLEM 3. Describe $m$-potent and $p$-central restricted colour Lie superalgebras represented as blocked matrices of directed graphs (see [13]).

\section{Potent and $p$-CEntral Restricted Lie algebras}

Let $\mathfrak{L}$ be a restricted Lie algebra over the field $\mathbb{F}$ of characteristic $p>0$. For a subset $S$ of $\mathfrak{L}$, we denote by $\langle S\rangle_{p}$ the restricted subalgebra generated by $S$. If $I$ is an ideal of $\mathfrak{L}$ then $I_{p}:=\langle I\rangle_{p}$ is a restricted ideal of $\mathfrak{L}$. By $S^{[p]^{k}}, k>0$, we denote the $\mathbb{F}$-vector subspace of $\mathfrak{L}$ spanned by the elements $x^{[p]^{k}}, x \in S$. The restricted Lie algebra $\mathfrak{L}$ is cyclic, if there exists $x \in \mathfrak{L}$ such that $\mathfrak{L}=\langle x\rangle_{p}$.

For a positive integer $i$ we denote by $\gamma_{i}(\mathfrak{L})$ the $i^{\text {th }}$ term of the lower central series of $\mathfrak{L}$. For a restricted Lie algebra $\mathfrak{L} \in \mathfrak{F}_{p}$, we denote by $\operatorname{cl}(\mathfrak{L})$ the nilpotency class of $\mathfrak{L}$, and by $e(\mathfrak{L})$ its exponent, that is, the minimum number $m \in \mathbb{N}_{0}$ such that $\mathfrak{L}^{[p]^{m}}=0$. The element $x \in \mathfrak{L}$ is called of exponent $k, k \in \mathbb{N}_{0}$, if and only if $\langle x\rangle_{p}$ is of exponent $k$. For an ideal $I$ of the Lie algebra $\mathfrak{L}$ we put $\left[I,{ }_{n} \mathfrak{L}\right]:=[\ldots[[I, \mathfrak{L}], \mathfrak{L}], \ldots, \mathfrak{L}]$, where $\mathfrak{L}$ appears in the latter expression $n$ times.

2.1. The Frattini ideal $\Phi(\mathfrak{L})$. Let $\mathfrak{L} \in \mathfrak{F}_{p}$. The restricted Lie ideal

$$
\Phi(\mathfrak{L}):=\mathfrak{L}^{[p]}+[\mathfrak{L}, \mathfrak{L}]
$$

will be called the Frattini ideal of $\mathfrak{L}$. For the convenience of the reader we state its well-known properties in the following proposition (see [21]).

Proposition 2.1. Let $\mathfrak{L} \in \mathfrak{F}_{p}$.

(a) $\Phi(\mathfrak{L})$ is the intersection of all restricted Lie ideals $I$ of $\mathfrak{L}$ of codimension 1 .

(b) If $S$ is a subset of $\mathfrak{L}$ whose image in $\mathfrak{L} / \Phi(\mathfrak{L})$ spans $\mathfrak{L} / \Phi(\mathfrak{L})$, then $\langle S\rangle_{p}=\mathfrak{L}$.

(c) Let $d(\mathfrak{L})$ denote the minimal number of generators of $\mathfrak{L}$ as restricted Lie algebra. Then $d(\mathfrak{L})=\operatorname{dim}_{\mathbb{F}}(\mathfrak{L} / \Phi(\mathfrak{L}))$.

(d) Let $J$ be a restricted ideal of $\mathfrak{L}$ being contained in $\Phi(\mathfrak{L})$. Then $\Phi(\mathfrak{L} / J)$ $=\Phi(\mathfrak{L}) / J$.

(e) Let $J$ be a 1-dimensional restricted Lie ideal of $\mathfrak{L}$ such that the short exact sequence $0 \rightarrow J \rightarrow \mathfrak{L} \rightarrow \mathfrak{L} / J \rightarrow 0$ is non-split. Then $J$ is contained in $\Phi(\mathfrak{L})$.

2.2. Potently embedded ideals. Let $p$ be odd and $m<p-1$. A restricted ideal $I$ of $\mathfrak{L} \in \mathfrak{F}_{p}$ is called m-potently embedded in $\mathfrak{L}$, if $[I, m \mathfrak{L}]$ is contained in $I^{[p]}$. If $p=2$, then $I$ is called 1-potently embedded in $\mathfrak{L}$, if $[I, \mathfrak{L}]$ is contained in $\left(I^{[2]}\right)^{[2]}$. A 1-potently embedded ideal will also be called a powerfully embedded ideal. Obviously, if $I$ is $m$ potently embedded in $\mathfrak{L}$, then $I^{[p]}$ is a restricted ideal of $L$. One has the following:

Lemma 2.2. Let $\mathfrak{L} \in \mathfrak{F}_{p}$ and let $I$ be a restricted ideal of $\mathfrak{L}$. 
(a) Let $p$ be odd and $m<p-1$. Then $I$ is $m$-potently embedded in $\mathfrak{L}$, if and only if $I /[I, p-1 \mathfrak{L}]_{p}$ is m-potently embedded in $\mathfrak{L} /[I, p-1 \mathfrak{L}]_{p}$. In this case one has $\left[I_{, p-1} \mathfrak{L}\right]_{p}=0$.

(b) Let $p=2$. Then $I$ is 1 -potently embedded in $\mathfrak{L}$, if and only if $I /\left[I,{ }_{3} \mathfrak{L}\right]_{2}$ is 1-potently embedded in $\mathfrak{L} /\left[I,{ }_{3} \mathfrak{L}\right]_{2}$. In this case one has $\left[I,{ }_{3} \mathfrak{L}\right]_{2}=0$.

PROOF: (a) Assume that $I /[I, p-1, \mathfrak{L}]_{p}$ is $m$-potently embedded in $\left.\mathfrak{L} /[I, p-1) \mathfrak{L}\right]_{p}$. It suffices to show that $\left[I_{, p-1} \mathfrak{L}\right]=0$. Suppose $\left[I_{, p-1} \mathfrak{L}\right] \neq 0$. By hypothesis,

$$
\left[I,{ }_{m} \mathfrak{L}\right]_{p}=\left(\left[I,_{m} \mathfrak{L}\right]_{p} \cap I^{[p]}\right)+\left[I,_{p-1} \mathfrak{L}\right]_{p}
$$

Put $J:=\left(\left[I,{ }_{m} \mathfrak{L}\right]_{p} \cap I^{[p]}\right)+\left[I,{ }_{p} \mathfrak{L}\right]_{p}$. Then $J$ is a restricted ideal of $\mathfrak{L}$, and by definition, $\left([I, m \mathfrak{L}]_{p} \cap I^{[p]}\right) \subseteq J \subseteq[I, m \mathfrak{L}]_{p}$. As $\mathfrak{L}$ is nilpotent and $\left.[I, p-1) \mathfrak{L}\right] \neq 0$, one has $[J, \mathfrak{L}]$ $\subseteq[I, p \mathfrak{L}] \subseteq[[I, m \mathfrak{L}], \mathfrak{L}]=\left[[I, m \mathfrak{L}]_{p}, \mathfrak{L}\right]$. In particular, $J \neq[I, m \mathfrak{L}]_{p}$. Since $\mathfrak{L}$ is finitedimensional and $p$-nilpotent, there exists a restricted ideal $K$ of $\mathfrak{L}$ such that $J \subseteq K$ $\subsetneq[I, m \mathfrak{L}]_{p}$, and $K$ has codimension 1 in $\left[I,,_{m} \mathfrak{L}\right]_{p}$. Put $\left[I,{ }_{m} \mathfrak{L}\right]_{p}=K+\mathbb{F}$. $x$ for a suitable $x \in[I, m \mathfrak{L}]_{p}$. Since every 1-dimensional left $\mathfrak{L}$-module is trivial, one concludes that $[[I, m \mathfrak{L}], \mathfrak{L}]_{p} \subseteq K$. By $(2.2)$ and as $m<p-1$, it follows that $\left[I,{ }_{m} \mathfrak{L}\right]_{p} \subseteq K$, a contradiction, and this yields the claim.

(b) The proof for $p=2$ can be obtained in a similar way by replacing the role of $I^{[p]}$ by $\left(I^{[2]}\right)^{[2]}$ and $[I, p-1, \mathfrak{L}]$ by $[I, 3 \mathfrak{L}]$.

For the reminder of this section we assume that $m$ is a positive integer satisfying $m<p-1$ for $p$ odd or $m=1$ in case $p=2$.

PROPOSITION 2.3. Let $\mathfrak{L} \in \mathfrak{F}_{p}$ and let $I$ and $J$ be two restricted ideals of $\mathfrak{L}$. If $I$ and $J$ are $m$-potently embedded in $\mathfrak{L}$, so are $[I, \mathfrak{L}]_{p}, I^{[p]},[I, J]_{p}$ and $I+J$.

Proof: Let $p$ be odd. First we show that $[I, \mathfrak{L}]$ is $m$-potently embedded. Without loss of generality we may assume that $\left[[I, \mathfrak{L}],_{p-1} \mathfrak{L}\right]=0$ (see Lemma 2.2(a)). Hence, for any $x \in I$ and $a \in \mathfrak{L}$, one has $(\operatorname{ad} x)^{p}(a)=0$, and thus $I^{[p]} \subseteq Z(\mathfrak{L})$. Since $I$ is $m$-potently embedded in $\mathfrak{L}$, this yields $\left[[I, \mathfrak{L}]_{p}, m \mathfrak{L}\right] \subseteq\left[I^{[p]}, \mathfrak{L}\right]=0$ and the claim follows.

Concerning $I^{[p]}$ we have already observed that $I^{[p]}$ is a restricted ideal of $\mathcal{L}$. By Lemma 2.2(a), we may assume that $\left[I^{[p]},{ }_{p-1} \mathfrak{L}\right]=0$. As $I$ is $m$-potently embedded in $\mathfrak{L}$, it follows that $\left[I_{m+p-1} \mathfrak{L}\right]=0$. Hence $\left[I^{[p]},{ }_{m} \mathfrak{L}\right]=0$, and $I^{[p]}$ is $m$-potently embedded in $\mathfrak{L}$.

Next consider $[I, J]_{p}$. As above we may assume that $\left[[I, J]_{p, p-1} \mathcal{L}\right]=0$. This forces $\left[I^{[p]}, J\right]=\left[I, J^{[p]}\right]=0$. Since $I$ and $J$ are $m$-potently embedded in $\mathcal{L}$, this implies that $[[I, m \mathfrak{L}], J] \subseteq\left[I^{[p]}, J\right]=0$ and $[[J, m \mathfrak{L}], I] \subseteq\left[I, J^{[p]}\right]=0$. By Jacobi's identity, one has $0=\left[[I, J]_{m} \mathfrak{L}\right]=\left[[I, J]_{p}, \mathfrak{L}\right]$, and thus $[I, J]_{p}$ is $m$-potently embedded in $\mathfrak{L}$.

Finally, for $I+J$ one has

$$
\left[I+J,_{m} \mathfrak{L}\right]=\left[I,_{m} \mathfrak{L}\right]+\left[J,_{m} \mathfrak{L}\right] \subseteq I^{[p]}+J^{[p]} \subseteq(I+J)^{[p]}
$$


therefore $I+J$ is $m$-potently embedded in $\mathfrak{L}$.

For $p=2$, the proof is analogous to the case $p$ odd using Lemma 2.2(b) and suitable modifications.

As a consequence of Proposition 2.3 one obtains the following corollary.

COROLlary 2.4. Any restricted Lie algebra $\mathfrak{L} \in \mathfrak{F}_{p}$ contains a unique maximal $m$-potently embedded restricted ideal.

A restricted $m$-potently embedded ideal $I$ of $\mathfrak{L}$ is obviously $m$-potent. If $\mathfrak{H}$ is a restricted subalgebra of $\mathfrak{L}$ and $\mathfrak{H} / I$ is cyclic, then $\mathfrak{H}$ is $m$-potent. Indeed, in this case there is $x \in \mathfrak{H}$ such that every element of $\mathfrak{H} / I$ is a linear combination of the elements $x^{[p]]^{i}}+I, i \in \mathbb{N}_{0}$. Consequently, $[\mathfrak{H}, \mathfrak{H}]=[I, \mathfrak{H}]$. As $I$ is $m$-potently embedded in $\mathfrak{L}$, one has

$$
\begin{array}{lll}
\gamma_{m+1}(\mathfrak{H}) \subseteq I^{[p]} & \subseteq \mathfrak{H}^{[p]} & \text { for } p \text { odd } \\
\gamma_{m+1}(\mathfrak{H}) \subseteq\left(I^{[2]}\right)^{[2]} & \subseteq\left(\mathfrak{H}^{[2]}\right)^{[2]} & \text { for } p=2 .
\end{array}
$$

The $m$-potency of a restricted Lie algebras is preserved by extension of the ground field. Furthermore, quotient Lie algebras and direct sums of $m$-potent restricted Lie algebras are $m$-potent as well. The following example shows that a restricted ideal of a $m$-potent restricted Lie algebra need not be $m$-potent.

EXAMPLE 2.5. Let $\mathfrak{L}$ be the Lie algebra over a field $\mathbb{F}$ of odd characteristic with $\mathbb{F}$-basis $\{x, y, z, v\}$ and with relations $[x, y]=z$ and $z, v \in Z(\mathfrak{L})$. The $p$-map $\mathfrak{L} \mathfrak{L}$ is given by

$$
x^{[p]}=y^{[p]}=z^{[p]}=0, \quad v^{[p]}=z .
$$

One has $[\mathfrak{L}, \mathfrak{L}]=\mathfrak{L}^{[p]}=\mathbb{F} . z$, and thus $\mathfrak{L}$ is powerful. For the restricted ideal $I:=\mathbb{F} \cdot x+\mathbb{F} \cdot y+\mathbb{F} . z$ one has $[I, I]=\mathbb{F} . z$, while $I^{[p]}=0$. Therefore, $I$ is not powerful.

THEOREM 2.6. Let $\mathfrak{L} \in \mathfrak{F}_{p}$ be an m-potent restricted Lie algebra.

(a) $\mathfrak{L}$ is nilpotent of class $\operatorname{cl}(\mathfrak{L}) \leqslant m+1$.

(b) For $i \geqslant 0$ the $\mathbb{F}$-vector space $\mathfrak{L}^{[p]^{i}}$ is a restricted ideal of $\mathfrak{L}$. Moreover, $\left(\mathfrak{L}^{\left[p^{p}\right.}\right)^{[p]^{\mathfrak{j}}}=\mathfrak{L}^{[p]^{[+j}}$.

(c) Let $\left\{b_{1}, \ldots, b_{r}\right\}$ be an $\mathbb{F}$-basis of $\mathfrak{L}$. Then $\mathfrak{L}^{[p]^{i}}=\sum_{1 \leqslant k \leqslant r} \mathbb{F} \cdot b_{k}^{[p]^{i}}$.

(d) If $\mathbb{F}$ is perfect, for every element $x$ of $\mathfrak{L}^{[p]^{i}}$ there exists $y \in \mathfrak{L}$ such that $y^{[p]^{i}}=x$.

Proof: (a) Let $p$ be odd. By Lemma 2.2(a), $\operatorname{cl}(\mathfrak{L}) \leqslant p-1$. For every $x, y \in \mathfrak{L}$ one has $\operatorname{ad} x^{[p]}(y)=0$ and thus $\mathfrak{L}^{[p]} \subseteq \mathrm{Z}(\mathfrak{L})$. Moreover, as $\mathfrak{L}$ is $m$-potent, $\gamma_{m+1}\left(\mathfrak{L} / \mathfrak{L}^{[p]}\right)=0$ and thus $\operatorname{cl}(\mathfrak{L}) \leqslant m+1$.

Let $p=2$. By Lemma $2.2(\mathrm{~b}), \operatorname{cl}(\mathfrak{L}) \leqslant 3$. One concludes that $\left(\operatorname{ad} x^{[2]}\right)^{2}(y)=0$ for every $x, y \in \mathfrak{L}$. Hence $\left(\mathfrak{L}^{[2]}\right)^{[2]} \subseteq Z(\mathfrak{L})$. Since $\mathfrak{L}$ is 1-potent, $\gamma_{2}\left(\mathfrak{L} /\left(\mathfrak{L}^{[2]}\right)^{[2]}\right)=0$ and therefore $\operatorname{cl}(\mathfrak{L}) \leqslant 2$. 
(b) By (a), the $\mathbb{F}$-vector subspace $\mathfrak{L}^{p]^{i}}$ of $\mathfrak{L}$ is contained in $Z(\mathfrak{L})$ for every $i>0$. This yields (b). Part (c) and (d) follow from the fact that ${ }_{-}^{[p]^{i}}: \mathfrak{L} \rightarrow Z(\mathfrak{L})$ is a $p$-semilinear map.

One has the following characterisation of powerful restricted Lie algebras.

Proposition 2.7. Let $\mathfrak{L} \in \mathfrak{F}_{p}$ be a restricted Lie algebra with $d:=d(\mathfrak{L})$.

(a) If $\mathfrak{L}$ is powerful, then $\mathfrak{L}$ is a sum of $d$ cyclic restricted Lie algebras.

(b) If $p \neq 2$, then $\mathfrak{L}$ is powerful, if and only if $\mathfrak{L}$ is the sum of $d$ cyclic restricted Lie algebras.

Proof: (a) Since $\mathfrak{L}$ is powerful, one has $\Phi(\mathfrak{L})=\mathfrak{L}^{[p]}$. Let $\pi_{\Phi}: \mathfrak{L} \rightarrow \mathfrak{L} / \Phi(\mathfrak{L})$ denote the canonical projection, and let $S=\left\{x_{1}, x_{2}, \ldots, x_{d}\right\}$ be a subset of $\mathfrak{L}$ such that $\pi_{\Phi}(S)$ is a basis of the $\mathbb{F}$-vector space $\mathfrak{L} / \mathfrak{L}^{[p]}$. Denote by $H:=\sum_{i=1}^{d}\left\langle x_{i}\right\rangle_{p}$ the sum of the cyclic restricted Lie algebras $\left\langle x_{i}\right\rangle_{p}$. By construction, one has $\pi_{\Phi}(H)=\pi_{\Phi}(\mathfrak{L})$. Hence $H+\mathfrak{L}^{[p]}=\mathfrak{L}$. As ${ }^{[p]}: \mathfrak{L} \rightarrow \mathfrak{L}^{[p]}$ is $p$-semilinear and $\mathfrak{L}^{[p]} \leqslant Z(\mathfrak{L})$, this implies $\mathfrak{L}^{[p]}=H^{[p]}+\mathfrak{L}^{[p]^{2}}$. Thus, by induction, $\mathfrak{L}^{[p]}=H^{[p]}$ and this yields the claim.

(b) Let $\mathfrak{L}=\sum_{i=1}^{d}\left\langle x_{i}\right\rangle_{p}$. The $\mathbb{F}$-subspace $\sum_{i=1}^{d}\left\langle x_{i}^{[p]}\right\rangle_{p}$ has codimension $d=d(\mathfrak{L})$ and is contained in $\operatorname{ker}\left(\pi_{\Phi}\right)$. Hence $\operatorname{ker}\left(\pi_{\Phi}\right)=\sum_{i=1}^{d}\left\langle x_{i}^{[p]}\right\rangle_{p}$. This implies $[\mathfrak{L}, \mathfrak{L}] \leqslant \Phi(\mathfrak{L}) \leqslant \mathfrak{L}^{[p]}$ and $\mathfrak{L}$ is powerful.

The following example shows that Proposition 2.7(b) does not hold in even characteristic:

EXAMPLE 2.8. Let $\mathfrak{H}$ be the 3-dimensional Heisenberg algebra over a field $\mathbb{F}$ of characteristic 2. Then $H$ has a basis $\{x, y, z\}$ with

$$
[x, y]=z, \quad[x, z]=[y, z]=0 .
$$

Consider the $p$-map on $\mathfrak{H}$ given by

$$
x^{[2]}=y^{[2]}=z, \quad z^{[2]}=0 .
$$

Then $d(\mathfrak{H})=2$ and $\mathfrak{H}=\langle x\rangle_{p}+\langle y\rangle_{p}$, but $\mathfrak{H}$ is not powerful.

The following property is useful for the characterisation of powerful restricted Lie algebras in terms of cohomological properties.

Proposition 2.9. Let $p$ be odd, and let $\mathfrak{L} \in \mathfrak{F}_{p}$ be a non-powerful restricted Lie algebra. Then there exists a restricted Lie ideal $J$ of $\mathfrak{L}$, such that

(i) $\mathfrak{L}^{[p]}$ is contained in $J$.

(ii) $J$ is contained in $\Phi(\mathfrak{L})$ and has codimension 1 . 
ProOF: The restricted Lie algebra $\mathfrak{L}$ is powerful, if and only if $\mathfrak{L} / \gamma_{p}(\mathfrak{L})_{p}$ is powerful (see Lemma 2.2(a)). Since $\gamma_{p}(\mathfrak{L})_{p}$ is contained in $\Phi(\mathfrak{L})$, we may therefore assume that $\gamma_{p}(\mathfrak{L})_{p}=0$ (see Proposition 2.1(d)). In particular, $\mathfrak{L}^{[p]}$ is a restricted Lie ideal contained in $Z(\mathfrak{L})$. Since $\mathfrak{L}$ is non-powerful, $\mathfrak{L}^{|p|}$ is properly contained in $\Phi(\mathfrak{L})$. Let $J$ be a maximal ideal being properly contained in $\Phi(\mathfrak{L})$ containing $\mathfrak{L}^{[p]}$. Then $J$ has the desired properties.

2.3. $p$-CENTRAL RESTRICTEd Lie algebras. For a restricted Lie algebra $\mathfrak{L}$ with $p$ map _ $[p]: \mathfrak{L} \rightarrow \mathfrak{L}^{[p]}$ we denote by $\mathfrak{L}_{[p]}$ the set of all zeros of $[p]$. Thus, $\mathfrak{L}$ is $p$-central, if and only if $\mathfrak{L}_{[p]} \subseteq Z(\mathfrak{L})$. If $\mathfrak{L}$ is a $p$-central restricted Lie algebra, $\mathfrak{L}_{[p]}$ is a restricted ideal. The property of $p$-centrality will be inherited on restricted subalgebras and is preserved by direct sums and extensions of the ground field. However, homomorphic images of $p$-central restricted Lie algebras need not be $p$-central. More precisely, any restricted Lie algebras is the homomorphic image of a $p$-central restricted Lie algebra.

PROPOSITION 2.10. Let $\mathfrak{L}$ be a restricted Lie algebra of dimension $n$ over a field $\mathbb{F}$ of characteristic $p>0$. Then there exists a $p$-central restricted Lie algebra $\tilde{\mathfrak{L}}$ such that $\operatorname{dim}_{\mathbb{F}}(\widetilde{\mathfrak{L}})=2 n$ and $\mathfrak{L}$ is isomorphic to $\tilde{\mathfrak{L}} / \widetilde{\mathfrak{L}}_{\{p\}}$ as a restricted Lie algebra.

ProOF: Let $\left\{x_{1}, \ldots, x_{n}\right\}$ be an $\mathbb{F}$-basis for $\mathfrak{L}$ and let $\mathfrak{B}$ be an Abelian $n$-dimensional Lie algebra over $\mathbb{F}$ with basis $\left\{y_{1}, \ldots, y_{n}\right\}$. Let $\widetilde{\mathfrak{L}}$ denote the Lie algebra $\mathfrak{L} \oplus \mathfrak{B}$ with $p$-map $\left[p^{\prime}\right]$ given by

$$
x_{1}^{\left[p^{\prime}\right]}=x_{1}^{[p]}+y_{1} ; \quad \cdots \quad x_{n}^{\left[p^{\prime}\right]}=x_{n}^{[p]}+y_{n} ; \quad y_{1}^{\left[p^{\prime}\right]}=\cdots=y_{n}^{\left[p^{\prime}\right]}=0 .
$$

Clearly, for $z=x+y \in \tilde{\mathfrak{L}}$ with $x=\sum_{i=1}^{n} \lambda_{i} x_{i} \in \mathfrak{L}$ and $y \in \mathfrak{B}$ one has

$$
z^{\left[p^{\prime}\right]}=\sum_{i=1}^{n} \lambda_{i}^{p} \cdot x_{i}^{[p]}+\sum_{i=1}^{n} \lambda_{i}^{p} \cdot y_{i} .
$$

The linear independence of the elements $x_{1}, \ldots, x_{n}, y_{1}, \ldots, y_{n}$ forces $\widetilde{\mathfrak{L}}_{[p]}=\mathfrak{B}$, and this yields the claim.

The following property which has been studied for finite groups in [5] yields a criterion for $p$-centrality in case that the nilpotency class is less than $p$.

PROPOSITION 2.11. Let $\mathfrak{L}$ be a nilpotent restricted Lie algebra over a field $\mathbb{F}$ of characteristic $p>0$ with $\operatorname{cl}(\mathfrak{L})<p$. Then $\mathfrak{L}$ is $p$-central, if and only if one has $[x, y]=0$ for every $x, y \in \mathfrak{L}$ satisfying $x^{[p]}=y^{[p]}$.

PROOF: Assume that $\mathfrak{L}$ is $p$-central. Since $\operatorname{cl}(\mathfrak{L})<p$, the $p$-map is $p$-semilinear. Hence $x^{[p]}=y^{[p]}$ forces $(x-y)^{[p]}=0$. This yields $x-y \in Z(\mathfrak{L})$, and thus $[x, y]=0$.

Conversely, suppose that for $x, y \in \mathfrak{L}, x^{[p]}=y^{[p]}$ implies $[x, y]=0$. Since $\operatorname{cl}(\mathfrak{L})<p$, for every $x \in \mathfrak{L}$ and $z \in \mathfrak{L}_{[p]}$, one has $(x+z)^{[p]}=x^{[p]}+z^{[p]}=x^{[p]}$. So, by hypothesis, $z \in \mathrm{Z}(\mathfrak{L})$ and this yields the claim. 
The following examples show that in contrast to the situation for finite groups (see [5]), one cannot drop the hypothesis on the nilpotency class.

EXAMPLE 2.12. Let $\mathbb{F}$ be a field of characteristic $p>0$. Let $\mathfrak{L}$ be the restricted $\mathbb{F}$ Lie algebra with basis $x, y, z, a_{1}, \ldots, a_{p-1}, b_{1}, \ldots, b_{p-1}$ subject to the following relations: $b_{i}, z \in Z(\mathfrak{L}), 1 \leqslant i \leqslant p-1$ and $[x, y]=a_{1},\left[x, a_{i}\right]=\left[a_{i}, a_{j}\right]=0$ for every $i, j<p$, $\left[a_{2}, y\right]=a_{i+1}$ for $i<p-1$ and $\left[a_{p-1}, y\right]=0$. In particular, $\operatorname{cl}(\mathfrak{L})=p$. The $p$-map is given by $x^{[p]}=0, y^{[p]}=z, z^{[p]}=0, a_{i}^{[p]}=b_{i}, b_{i}^{[p]}=0,1 \leqslant i \leqslant p-1$. A straightforward verification shows that any two elements of $\mathfrak{L}$ having the same image under the $p$-map commute. However, $x^{[p]}=0$ while $x \notin \mathrm{Z}(\mathfrak{L})$. Therefore, $\mathfrak{L}$ is not $p$-central.

EXAMPLE 2.13. Let $\mathfrak{M}$ be the restricted Lie algebra which coincides with $\mathcal{L}$ of Example 2.12 as $\mathbb{F}$-Lie algebra, but which $p$-map is given by $x^{[p]}=y^{[p]}=z, z^{[p]}=0, a_{i}^{[p]}=b_{i}$ and $b_{i}^{[p]}=0$ for $1 \leqslant i \leqslant p-1$. It is an easy exercise to verify that $\mathfrak{L}$ is $p$-central. However, $x^{[p]}=y^{[p]}$, but $[x, y] \neq 0$.

\section{The RESTRICTED ENVELOPING ALGEBRA OF POWERFUl RESTRICTEd LiE}

\section{A LGEBRAS}

Let $\mathcal{L}$ be a restricted Lie algebra over a field of characteristic $p>0$. By $u(\mathfrak{L})$ we shall denote the restricted universal enveloping algebra of $\mathcal{L}$, and by $\omega(\mathfrak{L})$ we shall denote the augmentation ideal of $\mathfrak{u}(\mathfrak{L})$, that is, $\omega(\mathfrak{L})$ is the kernel of the counit $\varepsilon: u(\mathfrak{L}) \rightarrow \mathbb{F}$ of the $\mathbb{F}$-Hopf algebra $u(\mathfrak{L})$. In particular, $\omega(\mathfrak{L})$ is the associative ideal generated by $\mathfrak{L}$ in $\mathfrak{u}(\mathfrak{L})$.

3.1. The nilpotency index of the aUgmentation ideal. It is well known (see $[20])$ that $\omega(\mathfrak{L})$ is nilpotent, if and only if $\mathfrak{L} \in \mathfrak{F}_{p}$. The nilpotency index $t(\mathfrak{u}(\mathfrak{L}))$ of $\omega(\mathfrak{L})$ is defined to be the smallest positive integer $k$ such that $\omega(\mathfrak{L})^{k}=0$. Relations beetwen the nilpotency index $t(\mathfrak{u}(\mathfrak{L}))$ of $\omega(\mathfrak{L})$ and the exponent $e(\mathfrak{L})$ of $\mathfrak{L}$ were studied in [21]: for example, it was shown that $p^{e(\mathfrak{L})} \leqslant t(\mathfrak{u}(\mathfrak{L}))$ for all $\mathfrak{L} \in \mathfrak{F}_{p}$. For powerful restricted Lie algebras one has also the following.

PROPOSITION 3.1. Let $\mathfrak{L}$ be a powerful restricted Lie algebra over a field $\mathbb{F}$ of characteristic $p>0$. Then one has

$$
t(\mathrm{u}(\mathfrak{L})) \leqslant 1+d(\mathfrak{L}) \cdot\left(p^{e(\mathfrak{L})}-1\right) .
$$

Moreover, equality holds in (3.1), if and only if every element $x \in \mathfrak{L} \backslash \mathfrak{L}^{[p]}$ is of exponent $e(\mathfrak{L})$.

Proof: Put

$$
\mathfrak{D}_{1}(\mathfrak{L}):=\mathfrak{L}, \quad \mathfrak{D}_{m}(\mathfrak{L}):=\left\langle\mathfrak{D}_{[m / p]}(\mathfrak{L})^{[\mathfrak{p}]}\right\rangle_{p}+\left[\mathfrak{L}, \mathfrak{D}_{m-1}(\mathfrak{L})\right] \text { for } m>1 .
$$


By $[21]$, one has

$$
t(u(\mathfrak{L}))=1+(p-1) \cdot \sum_{n \geqslant 1} n \cdot d_{n}
$$

where $d_{n}:=\operatorname{dim}_{F}\left(\mathfrak{D}_{n}(\mathfrak{L}) / \mathfrak{D}_{n+1}(\mathfrak{L})\right)$. By Theorem 2.6 and induction, one concludes easily that

$$
\mathfrak{D}_{n}(\mathfrak{L})=\mathfrak{L}^{\left[p^{j(n)}\right.}
$$

where $k(n):=\left\lceil\log _{p} n\right\rceil$. This yields

$$
d_{n}= \begin{cases}\operatorname{dim}_{\mathbb{F}}\left(\mathfrak{L}^{[p]^{i}} / \mathfrak{L}^{[p]^{i+1}}\right) & \text { if } n=p^{i} \text { with } 0 \leqslant i<e(\mathfrak{L}) \\ 0 & \text { otherwise. }\end{cases}
$$

Formula (3.3) implies that

$$
t(\mathfrak{u}(\mathfrak{L}))=1+(p-1) \cdot \sum_{i=0}^{e(\mathfrak{L})-1} p^{i} \cdot \operatorname{dim}_{\mathbb{F}}\left(\mathfrak{L}^{[p]^{i}} / \mathfrak{L}^{[p]^{i+1}}\right) .
$$

Moreover, by Theorem 2.6, $\operatorname{dim}_{\mathbb{F}}\left(\mathfrak{L}^{[p]^{i}} / \mathfrak{L}^{[p]^{i+1}}\right) \leqslant d(\mathfrak{L})$ which yields (3.1). One has equality in $(3.1)$, if and only if $\operatorname{dim}_{\mathbb{F}}\left(\mathfrak{L}^{[p\}^{i}} / \mathfrak{L}^{[p]^{i+1}}\right)=d(\mathfrak{L})$ for all $i=0, \ldots, e(\mathfrak{L})-1$. By Theorem 2.6, this is equivalent to the property that every element $x \in \mathfrak{L} \backslash \mathfrak{L}^{[p]}$ is of exponent $e(\mathfrak{L})$.

3.2. The Lie DeRIVEd Length. Let $\mathfrak{A}$ be any associative $\mathbb{F}$-algebra with unit. The associative $\mathbb{F}$-algebra $\mathfrak{A}$ can be regarded as an $\mathbb{F}$-Lie algebra via the Lie commutator $[x, y]=x y-y x, x, y \in \mathfrak{A}$. The Lie derived series $\delta^{[n]}(\mathfrak{A})$ and the strong Lie derived series $\delta^{(n)}(\mathfrak{A})$ of $\mathfrak{A}$ are given by

$$
\begin{aligned}
\delta^{[0]}(\mathfrak{A}): & =\delta^{(0)}(\mathfrak{A})=\mathfrak{A} \\
\delta^{[n]}(\mathfrak{A}): & =\left[\delta^{[n-1]}(\mathfrak{A}), \delta^{[n-1]}(\mathfrak{A})\right] \\
\delta^{(n)}(\mathfrak{A}): & =\left[\delta^{(n-1)}(\mathfrak{A}), \delta^{(n-1)}(\mathfrak{A})\right] \mathfrak{A}
\end{aligned}
$$

The associative $\mathbb{F}$-algebra $\mathfrak{A}$ is called Lie solvable (respectively strongly Lie solvable), if $\delta^{[n]}(\mathfrak{A})=0$ (respectively $\delta^{(n)}(\mathfrak{A})=0$ ) for some $n>0$. The smallest such number $n$ is called the Lie derived length (respectively strong Lie derived length) and will be denoted by $\operatorname{dl}_{\text {Lie }}(\mathfrak{A})$ (respectively $\mathrm{dl}^{\text {Lie }}(\mathfrak{A})$ ). Obviously, if $\mathfrak{A}$ is strongly Lie solvable, then $\mathfrak{A}$ is Lie solvable and $\mathrm{dl}_{\text {Lie }}(\mathfrak{A}) \leqslant \mathrm{dl}^{\mathrm{Lie}}(\mathfrak{A})$.

Let $\mathfrak{L}$ be a finite-dimensional restricted Lie algebra over a field $\mathbb{F}$ of characteristic $p>0$. Under the assumption that $\mathbb{F}$ is of odd characteristic, Riley and Shalev proved in [20] that $\mathfrak{u}(\mathfrak{L})$ is Lie solvable, if and only if $\mathfrak{L}_{p}^{\prime}:=[\mathfrak{L}, \mathfrak{L}]_{p}$ is $p$-nilpotent. In [23] it was shown that - without any restriction on the ground field $-\mathfrak{u}(\mathfrak{L})$ is strongly Lie solvable, 
if and only if $\mathfrak{L}_{p}^{\prime}$ is $p$-nilpotent. However, for such a restricted Lie algebra it can happen that $\mathrm{dl}_{\text {Lie }}(\mathfrak{u}(\mathfrak{L})) \neq \mathrm{dl}^{\mathrm{Lie}}(\mathfrak{u}(\mathfrak{L}))$. Apart from the results in $[\mathbf{2 2}, \mathbf{2 3}, \mathbf{2 5}]$, very little is known about the Lie derived lengths of the $\mathbb{F}$-algebra $\mathfrak{u}(\mathfrak{L})$. For powerful restricted Lie algebras one has the following property.

Proposition 3.2. Let $\mathfrak{L}$ be a restricted Lie algebra over a field $\mathbb{F}$ of characteristic $p>0$. If $\mathfrak{L}$ is powerful, then

$$
\begin{aligned}
\min \left(\left\lceil\log _{2}\left(p^{e\left(\mathfrak{L}_{p}^{\prime}\right)}+1\right)\right\rceil, p-1\right) & \leqslant \operatorname{dl}_{\text {Lie }}(u(\mathfrak{L})) \\
& \leqslant \mathrm{dl}^{\mathrm{Lie}}(u(\mathfrak{L})) \leqslant\left\lceil\log _{2}\left(2+d\left(\mathfrak{L}_{p}^{\prime}\right) \cdot\left(p^{e\left(\mathfrak{E}_{p}^{\prime}\right)}-1\right)\right)\right\rceil .
\end{aligned}
$$

Proof: By [23, Lem.2] and Proposition 3.1, one has

$$
\mathrm{dl}^{\mathrm{Lie}}(\mathfrak{u}(\mathfrak{L})) \leqslant\left\lceil\log _{2}\left(2+d\left(\mathcal{L}_{p}^{\prime}\right) \cdot\left(p^{e\left(\mathcal{L}_{p}^{\prime}\right)}-1\right)\right)\right\rceil .
$$

It remains to show, that if $\mathrm{dl}_{\text {Lie }}(u(\mathfrak{L}))<p$, then $\mathrm{dl}_{\text {Lie }}(u(\mathfrak{L})) \geqslant\left\lceil\log _{2}\left(p^{e\left(\mathcal{L}_{p}^{\prime}\right)}+1\right)\right\rceil$. If $\mathfrak{L}$ is Abelian, the claim is trivial. Assume that $\mathfrak{L}$ is non-Abelian, that is, $\operatorname{cl}(\mathfrak{L})=2$. By Theorem $A, \mathfrak{L}_{p}^{\prime}$ is Abelian. Consequently, there exist two non-commuting elements $a, b \in \mathfrak{L}$, such that $z:=[a, b]$ is of exponent $e\left(\mathfrak{L}_{p}^{\prime}\right)$. We claim that $a^{h} z^{2^{m}-1}, b^{k} z^{2^{m}-1}$ $\in \delta^{[m]}(\mathfrak{u}(\mathfrak{L}))$ for every non-negative integer $m$ and for every $0 \leqslant h, k \leqslant p-m-1$. We proceed by induction on $m$. For $m=0$, the claim is trivial. Assume that by induction, one has $a^{h+1} z^{2^{m-1}-1} \in \delta^{[m-1]}(\mathfrak{u}(\mathfrak{L}))$ and $b z^{2^{m-1}-1} \in \delta^{[m-1]}(\mathfrak{u}(\mathfrak{L}))$. As $z$ centralises $a$ and $b$, the Leibnitz rule implies that

$$
\left[a^{h+1}, b\right]=\sum_{i=1}^{h+1} a^{i-1}[a, b] a^{h-i+1}=\sum_{i=1}^{h+1} a^{h} z=(h+1) a^{h} z .
$$

In particular,

$$
\left[a^{h+1} z^{2^{m-1}-1}, b z^{2^{m-1}-1}\right]=\left[a^{h+1}, b\right] z^{2^{m}-2}=(h+1) a^{h} z^{2^{m}-1} .
$$

As $0<h+1<p$, one concludes that $a^{h} z^{2^{m}-1} \in \delta^{[m]}(u(\mathcal{L}))$, and a similar argument shows that $b^{k} z^{2^{m}-1} \in \delta^{[m]}(\mathfrak{u}(\mathfrak{L}))$. This yields the claim. The Poincaré-Birkhoff-Witt theorem for restricted universal enveloping algebras (see [26, Chapter 2, Theorem 5.1]) implies that for $2^{m}-1<p^{e\left(\mathfrak{L}_{p}^{\prime}\right)}$, the element $z^{2^{m}-1}$ is non-trivial. The claim has shown that for $0 \leqslant m \leqslant p-1$ the element $z^{2^{m}-1}$ is contained in $\delta^{[m]}(u(\mathfrak{L}))$, completing the proof of the proposition.

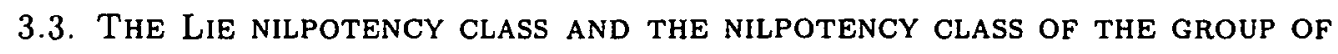
UNITs. Let $\mathbb{F}$ be a field, and let $\mathfrak{A}$ be an associative $\mathbb{F}$-algebra with unit. One calls $\mathfrak{A}$ Lie nilpotent, if $\mathfrak{A}$ is nilpotent as $\mathbb{F}$-Lie algebra. In this case we denote by $\mathrm{cl}_{\text {Lie }}(\mathfrak{A})$ the Lie nilpotency class of $\mathfrak{A}$. Put $\mathfrak{A}^{(1)}:=\mathfrak{A}$ and $\mathfrak{A}^{(n+1)}=\left[\mathfrak{A}^{(n)}, \mathfrak{A}^{(\mathfrak{n})}\right] \mathfrak{A}, n \geqslant 2$. One says that $\mathfrak{A}$ is strongly Lie nilpotent, if $\mathfrak{A}^{(n)}=0$ for some $n$. In this case one calls the minimal 
non-negative integer $\mathrm{cl}^{\mathrm{Lie}}(\mathfrak{A}):=m$ satisfying $\mathfrak{A}^{(m+1)}=0$ the strong Lie nilpotency class of $\mathfrak{A}$.

In [20], Riley and Shalev proved that if $\mathfrak{L}$ is a restricted Lie algebra over a field $\mathbb{F}$ of characteristic $p>0$, then $\mathfrak{u}(\mathfrak{L})$ is Lie nilpotent, if and only it is strongly Lie nilpotent. Moreover, this happens precisely when $\mathfrak{L}$ is nilpotent and $\mathfrak{L}_{p}^{\prime} \in \mathfrak{F}_{p}$. They also showed that $\operatorname{cl}_{\text {Lie }}(\mathfrak{u}(\mathfrak{L}))=\operatorname{cl}^{\text {Lie }}(\mathfrak{u}(\mathfrak{L}))$ provided $p>3$, while it is unknown whether this equality holds in the exceptional cases $p=2,3$ as well. In [24] it was shown, that if $\mathfrak{L} \in \mathfrak{F}_{p}$ and $\mathfrak{L}_{p}^{\prime}$ is cyclic, then $\operatorname{cl}_{\text {Lie }}(\mathfrak{u}(\mathfrak{L}))=\operatorname{cl}^{\mathrm{Lie}}(\mathfrak{u}(\mathfrak{L}))=p^{\operatorname{dim}_{\mathbb{R}} \mathcal{L}_{p}^{\prime}}$. Here we prove the following result:

Proposition 3.3. Let $\mathfrak{L} \in \mathfrak{F}_{p}$. If $\mathfrak{L}_{p}^{\prime}$ is powerfully embedded in $\mathfrak{L}$, then

$$
p^{e\left(\mathfrak{L}_{p}^{\prime}\right)} \leqslant \operatorname{cl}_{\text {Lie }}(\mathfrak{u}(\mathfrak{L})) \leqslant \mathrm{cl}^{\text {Lie }}(\mathfrak{u}(\mathfrak{L})) \leqslant 1+d\left(\mathfrak{L}_{p}^{\prime}\right) \cdot\left(p^{e\left(\mathfrak{L}_{p}^{\prime}\right)}-1\right) .
$$

Proof: From [24, Theorem 1] and Theorem 2.6 it follows that $\mathrm{cl}_{\mathrm{Lie}}(\mathfrak{u}(\mathfrak{L})) \geqslant p^{e\left(\mathfrak{L}_{p}^{\prime}\right)}$. Consider the chain of restricted ideals of $\mathfrak{L}$ defined recursively by

$$
\begin{aligned}
\mathfrak{D}_{1}(\mathfrak{L}): & =\mathfrak{L}, \quad \mathfrak{D}_{2}(\mathfrak{L}):=\mathfrak{L}_{p}^{\prime}, \\
\mathfrak{D}_{m+1}(\mathfrak{L}): & =\left\langle\mathfrak{D}_{([(m+p) / p])}(\mathfrak{L})^{(p)}\right\rangle_{p}+\left[\mathfrak{D}_{(m)}(\mathfrak{L}), \mathfrak{L}\right], \quad m \geqslant 2 .
\end{aligned}
$$

According to [21], one has

$$
\mathrm{cl}^{\mathrm{Lie}}(\mathbf{u}(\mathfrak{L}))=1+(p-1) \cdot \sum_{m \geqslant 1} m \cdot d_{(m+1)},
$$

where $d_{(m)}:=\operatorname{dim}_{\mathbf{F}}\left(\mathfrak{D}_{(m)}(\mathfrak{L}) / \mathfrak{D}_{(m+1)}(\mathfrak{L})\right)$. As $\mathfrak{L}_{p}^{\prime}$ is powerfully embedded in $\mathfrak{L}$, Proposition 2.3 and Theorem 2.6 imply that for $n>1$ one has

$$
\mathfrak{D}_{(n)}(\mathfrak{L})=\left(\mathfrak{L}_{p}^{\prime}\right)^{[p]^{h(n)}}
$$

where $h(n):=\left\lceil\log _{p}(n-1)\right\rceil$. From this identity one concludes that for $n \geqslant 2$

$$
d_{(n)}= \begin{cases}\operatorname{dim}_{\mathbb{F}}\left(\left(\mathfrak{L}_{p}^{\prime}\right)^{[p]^{i}} /\left(\mathfrak{L}_{p}^{\prime}\right)^{[p]^{i+1}}\right) & \text { if } n=p^{i}+1 \text { with } 0 \leqslant i<e\left(\mathfrak{L}_{p}^{\prime}\right), \\ 0 & \text { otherwise. }\end{cases}
$$

From formula (3.14) one deduces that

$$
\operatorname{cl}^{\mathrm{Lie}}(\mathrm{u}(\mathfrak{L}))=1+(p-1) \cdot \sum_{n=0}^{e\left(\mathfrak{L}_{p}^{\prime}\right)-1} p^{n} \cdot \operatorname{dim}_{\mathbb{F}}\left(\left(\mathfrak{L}_{p}^{\prime}\right)^{[p]^{n}} /\left(\mathfrak{L}_{p}^{\prime}\right)^{[p]^{n+1}}\right) .
$$

As in Proposition 3.1, this yields $\mathrm{cl}^{\mathrm{Lie}}(\mathfrak{u}(\mathfrak{L})) \leqslant 1+d\left(\mathfrak{L}_{p}^{\prime}\right) \cdot\left(p^{e\left(\mathfrak{L}_{p}^{\prime}\right)}-1\right)$.

For an associative $\mathbb{F}$-algebra $\mathfrak{A}$ with unit, we denote by $\mathfrak{A}^{*}$ the group of units of 2. Let $\operatorname{cl}(G)$ denote the nilpotency class of the nilpotent group $G$. If $\mathfrak{L} \in \mathfrak{F}_{p}$, then $\omega(\mathfrak{L})$ is nilpotent and $\mathfrak{u}(\mathfrak{L})^{*}=\mathbb{F}^{*} \times(1+\omega(\mathfrak{L}))$. Hence, $\mathfrak{u}(\mathfrak{L})^{*}$ is nilpotent and $\operatorname{cl}\left(\mathfrak{u}(\mathfrak{L})^{*}\right)$ $=\operatorname{cl}(1+\omega(\mathfrak{L}))$. According to a result of $\mathrm{Du}$ (see [8]), if an associative $\mathbb{F}$-algebra $\mathfrak{T}$ is radical, that is, $\mathfrak{T}$ coincides with its Jacobson radical, and Lie nilpotent, then $\operatorname{cl}_{\text {Lie }}(\mathfrak{T})$ coincides with the nilpotency class of the adjoint group $\mathfrak{T}^{\circ}=1+\mathfrak{T}$. As a consequence one obtains the following: 
COROLlaRY 3.4. Let $\mathfrak{L} \in \mathfrak{F}_{p}$. If $\mathfrak{L}_{p}^{\prime}$ is powerfully embedded in $\mathfrak{L}$, then

$$
p^{e\left(\mathfrak{L}_{p}^{\prime}\right)} \leqslant \operatorname{cl}\left(u(\mathfrak{L})^{*}\right) \leqslant 1+d\left(\mathcal{L}_{p}^{\prime}\right) \cdot\left(p^{e\left(\mathcal{L}_{p}^{\prime}\right)}-1\right) .
$$

\section{COHOMOLOGY FOR RESTRICTED LIE ALGEBRAS}

Let $\mathfrak{L}$ be a restricted Lie algebra and let $\mathfrak{u}(\mathfrak{L})$ denote its restricted universal enveloping $\mathbb{F}$-algebra. The $k^{\text {th }}$-cohomology group with coefficients in the left $\mathfrak{L}$-module $M$ is given by

$$
H^{k}(\mathfrak{L}, M):=\operatorname{Ext}_{\mathfrak{u}(\mathfrak{L})}^{k}(\mathbb{F}, M)
$$

where $\mathbb{F}$ denotes the trivial left $\mathfrak{L}$-module. Cup-product

$$
-\cup_{-}: H^{\bullet}(\mathfrak{L}, \mathbb{F}) \times H^{\bullet}(\mathfrak{L}, \mathbb{F}) \longrightarrow H^{\bullet}(\mathfrak{L}, \mathbb{F}),
$$

which coincides with the Yoneda composition of Ext-groups, gives $H^{\bullet}(\mathfrak{L}, \mathbb{F})$ naturally the structure of a graded commutative $\mathbb{F}$-algebra. Moreover, every homomorphism $\phi: \mathfrak{L} \rightarrow \mathfrak{M}$ induces a homomorphism of graded commutative $\mathbb{F}$-algebras $\phi^{\bullet}: H^{\bullet}(\mathfrak{M}, \mathbb{F}) \rightarrow H^{\bullet}(\mathfrak{L}, \mathbb{F})$. The reduced cohomology $\mathbb{F}$-algebra of the restricted Lie algebra $\mathfrak{L}$ is given by

$$
H^{\bullet}(\mathfrak{L}, \mathbb{F})_{\mathrm{red}}:=H^{\bullet}(\mathfrak{L}, \mathbb{F}) / \operatorname{nil}\left(H^{\bullet}(\mathfrak{L}, \mathbb{F})\right),
$$

where $\operatorname{nil}\left(H^{\bullet}(\mathfrak{L}, \mathbb{F})\right)$ denotes the graded ideal of nilpotent elements of the graded $\mathbb{F}$ algebra $H^{\bullet}(\mathcal{L}, \mathbb{F})$. Certainly, one of the most striking result on the cohomology of finitedimensional restricted Lie algebras is the theorem of Jantzen (see [11]). It states that if $\mathbb{F}$ is an algebraically closed field of characteristic $p, p$ odd, then $H^{\bullet}(\mathfrak{L}, \mathbb{F})_{\text {red }}$ can be identified with the rational functions on the algebraic set $\mathfrak{L}_{[p]}=\left\{x \in \mathfrak{L} \mid x^{[p]}=0\right\}$ generated as $\mathbb{F}$-algebra in degree 2. One can think of this theorem as the analogue of Quillen's theorem which describes $H^{\bullet}\left(G, \mathbb{F}_{p}\right)$ of a finite group $G$ up to $F$-isomorphism (see [18]).

4.1. Powerful restricted Lie algebras. If $p$ is odd, one can characterise powerful restricted Lie algebras in the class $\mathfrak{F}_{p}$ in the same way as powerful pro- $p$ groups (see $[\mathbf{2 7}$, Theorem 5.1.6]).

THEOREM 4.1. Let $p$ be odd and let $\mathfrak{L} \in \mathfrak{F}_{p}$. Then the following are equivalent:

(i) $\mathfrak{L}$ is powerful.

(ii) The mapping $\beta_{\mathfrak{L}}: H^{1}(\mathfrak{L}, \mathbb{F}) \wedge H^{1}(\mathfrak{L}, \mathbb{F}) \rightarrow H^{2}(\mathfrak{L}, F)$ induced by cup-product is injective.

The proof of Theorem 4.1 makes use of the following simple fact. 
FACT 4.2. Let $p$ be odd, and let $\mathfrak{A}$ be a finite-dimensional Abelian restricted Lie algebra with trivial $p$-map. Let $\eta \in H^{2}(\mathfrak{A}, F)$ and let

$$
\mathbf{s}_{\eta}: \quad 0 \longrightarrow \mathbb{F} \longrightarrow \mathfrak{A}_{\eta} \stackrel{\tau_{\eta}}{\longrightarrow} \mathfrak{A} \longrightarrow 0
$$

denote the corresponding short exact sequence of restricted Lie algebras (see [10]). Then one has $\mathfrak{A}_{\eta}^{[p]}=0$, if and only if $\eta \in \operatorname{im}\left(\beta_{\mathfrak{A}}\right)$.

PROOF: As $p$ is odd, the $p$-map on $\mathfrak{A}_{\eta}$ induces a $p$-semilinear map $\psi(\eta) \in \operatorname{Hom}_{F}^{p}(\mathfrak{A}, \mathbb{F})$ of degree $p$. This yields a short exact sequence

$$
0 \longrightarrow H^{\mathfrak{l}}(\mathfrak{A}, \mathbb{F}) \wedge H^{1}(\mathfrak{A}, \mathbb{F}) \stackrel{\beta_{\mathfrak{a}}}{\longrightarrow} H^{2}(\mathfrak{A}, \mathbb{F}) \stackrel{\psi}{\longrightarrow} \operatorname{Hom}_{\mathbf{F}}^{p}(\mathfrak{A}, \mathbb{F}) \longrightarrow 0,
$$

which implies the claim.

PROOF: [Proof of Theorem 4.1] Let $\pi: \mathfrak{L} \rightarrow \mathfrak{A}, \mathfrak{A}:=\mathfrak{L} / \Phi(\mathfrak{L})$, denote the canonical projection. One has a commutative diagram

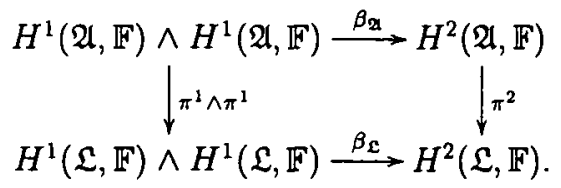

Moreover, $\pi^{1} \wedge \pi^{1}$ is an isomorphism, and $\beta_{\mathfrak{A}}$ is injective. For $\eta \in H^{2}(\mathfrak{A}, \mathbb{F})$, let $\mathfrak{L}_{\eta}$ denote the pull back of the mappings $\mathfrak{L} \rightarrow \mathfrak{A} \leftarrow \mathfrak{A}_{\eta}$, that is, one has a commutative diagram

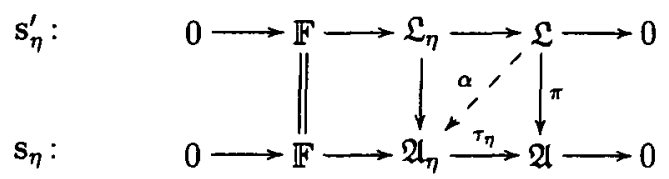

If $\mathbf{s}_{\eta}^{\prime}$ is split, there exists a mapping $\alpha$ making the diagram (4.7) commute. On the other hand, $\mathfrak{L}_{\eta}$ is the pull back of the mappings $\pi$ and $\tau_{n}$. Hence the existence of the mapping $\alpha$ in (4.7) implies that $\mathbf{s}_{\eta}^{\prime}$ is split. If $\eta \neq 0, \mathbf{s}_{\eta}$ is a Frattini extension (see Proposition 2.1(e)), and therefore, a mapping $\alpha$ making (4.7) commute must be surjective (see Proposition $2.1(\mathrm{~b}))$.

Let $\mathfrak{L}$ be powerful. Let $\xi^{\prime} \in H^{1}(\mathfrak{L}, \mathbb{F}) \wedge H^{1}(\mathfrak{L}, \mathbb{F}), \xi^{\prime} \neq 0$, and assume that $\beta_{\mathfrak{L}}\left(\xi^{\prime}\right)=0$. Let $\xi \in H^{1}(\mathfrak{A}, \mathbb{F}) \wedge H^{1}(\mathfrak{A}, \mathbb{F})$ such that $\left(\pi^{1} \wedge \pi^{1}\right)(\xi)=\xi^{\prime}$. Hence $\beta_{\mathfrak{A}}(\xi) \neq 0$ and $\mathbf{s}_{\beta_{\mathfrak{A}}(\xi)}$ is a Frattini extension. The commutativity of the diagram (4.6) and the previously mentioned remark imply that there exists a surjective map $\alpha: \mathfrak{L} \rightarrow \mathfrak{A}_{\beta_{\mathfrak{a}}(\xi)}$ making the diagram (4.7) commute for $\eta:=\beta_{\mathfrak{A}}(\xi)$. However, by Fact 4.2 , one has $\mathfrak{A}_{\beta_{\mathfrak{a}}(\xi)}^{[p]}=0$. Hence $\mathfrak{A}_{\beta_{\mathfrak{a}(\xi)}}$ is not powerful. On the other hand, as a homomorphic image of $\mathfrak{L}$ the restricted Lie algebra $\mathfrak{A}_{\beta_{\mathbf{a}}(\xi)}$ must be powerful, a contradiction. This yields the implication (i) $\Rightarrow$ (ii).

Let $\beta_{\mathfrak{L}}$ be injective, and assume that $\mathfrak{L}$ is not powerful. Hence there exists a restricted ideal $J$ of $\mathfrak{L}$ such that $\mathfrak{L}^{[p]} \subseteq J \subsetneq \Phi(\mathfrak{L})$ and $J$ has codimension 1 in $\Phi(\mathfrak{L})$ (see Proposition 
2.9). Let $\mathfrak{H}:=\mathfrak{L} / J$ and let

$$
\mathrm{s}: \quad 0 \longrightarrow \mathbb{F} \longrightarrow \mathfrak{H} \stackrel{\sigma}{\longrightarrow} \mathfrak{A} \longrightarrow 0
$$

denote the canonical short exact sequence. By construction, $s$ is non-split and $\mathfrak{H}^{[p]}=0$. Hence by Fact 4.2 , there exists an element $\xi \in H^{1}(\mathfrak{A}, \mathbb{F}) \wedge H^{1}(\mathfrak{A}, \mathbb{F}), \xi \neq 0$, such that $\mathbf{s}=\mathbf{s}_{\theta_{\mathbf{a}}(\xi)}$. From the commutative diagram

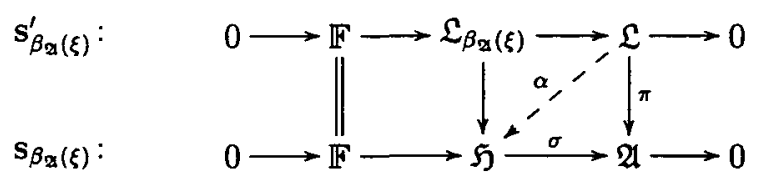

one concludes that $\beta_{\mathfrak{L}}\left(\left(\pi^{1} \wedge \pi^{1}\right)(\xi)\right)=0$. Hence $\beta_{\mathfrak{L}}$ is not injective, a contradiction, and this completes the proof of the theorem.

4.2. Cohomology for $p$-CENTRAL Restricted Lie algebras. Let $\mathfrak{L}$ be a finitedimensional $p$-central restricted Lie algebra. For such a restricted Lie algebra one has a surjective homomorphism

$$
\rho: \mathfrak{L}_{[p]} \oplus \mathfrak{L} \longrightarrow \mathfrak{L}, \quad \rho(z, x):=z+x .
$$

Applying Künneth' theorem one obtains a mapping

$$
\Delta_{\mathfrak{L}}:=(\operatorname{red} \otimes \mathrm{id}) \circ \rho^{\bullet}: H^{\bullet}(\mathfrak{L}, \mathbb{F}) \rightarrow H^{\bullet}\left(\mathfrak{L}_{[p]}, \mathbb{F}\right)_{\mathrm{red}} \otimes H^{\bullet}(\mathfrak{L}, \mathbb{F}),
$$

which gives $H^{\bullet}(\mathfrak{L}, \mathbb{F})$ the structure of a left $H^{\bullet}\left(\mathfrak{L}_{[p]}, \mathbb{F}\right)_{\text {red }}$-comodule algebra. The Hopf algebra structure on $H^{\bullet}\left(\mathfrak{L}_{[p]}, \mathbb{F}\right)_{\text {red }}$ is induced by the mapping $\Delta_{\mathfrak{L}_{[p]}}$ (see [17]). Using this additional structure one deduces the following.

THEOREM 4.3. Let $p$ be odd and let $\mathfrak{L}$ be a finite-dimensional restricted Lie algebra. Then one has an isomorphism of graded commutative $\mathbb{F}$-algebras

$$
H^{\bullet}(\mathfrak{L}, \mathbb{F}) \simeq C^{\bullet} \otimes S^{\bullet}\left(\mathfrak{L}_{[p]}^{*}\right)
$$

where $S^{\bullet}\left(\mathcal{L}_{[p]}^{*}\right)$ is generated in degree 2 and $C^{\bullet}$ is a finite-dimensional graded commutative $\mathbb{F}$-algebra. In particular, $H^{\bullet}(\mathfrak{L}, \mathbb{F})$ is a graded commutative Cohen-Macaulay $\mathbb{F}$-algebra.

PROOF: Let $\iota: \mathfrak{L}_{[p]} \rightarrow \mathfrak{L}$ denote the canonical map. The theorem of Jantzen implies that the reduced restriction map

$$
j^{\bullet}:=\operatorname{red} \circ \iota^{\bullet}: H^{\bullet}(\mathfrak{L}, \mathbb{F}) \longrightarrow H^{\bullet}\left(\mathfrak{L}_{[p]}, \mathbb{F}\right)_{\mathrm{red}}
$$

is surjective. Thus [28, Theorem 3.1] implies that one has an isomorphism of $\mathbb{F}$-algebras

$$
H^{\bullet}(\mathfrak{L}, \mathbb{F}) \simeq C^{\bullet} \otimes H^{\bullet}\left(\mathfrak{L}_{[p]}, \mathbb{F}\right)_{\text {red }}
$$




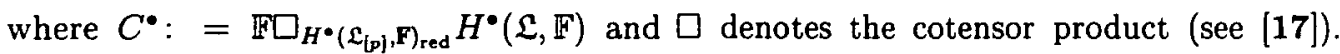
Moreover, since $H^{\bullet}(\mathfrak{L}, \mathbb{F})$ is a finitely generated graded commutative $\mathbb{F}$-algebra (see [12, Section 1.11, Proposition]), $C^{\bullet}$ is also finitely generated. By Jantzen's theorem,

$$
\iota_{\text {red }}: H^{\bullet}(\mathfrak{L}, \mathbb{F})_{\text {red }} \longrightarrow H^{\bullet}\left(\mathfrak{L}_{\{p\}}, \mathbb{F}\right)_{\text {red }}
$$

is an isomorphism. This implies that the augmentation ideal $\omega\left(C^{\bullet}\right)$ of $C^{\bullet}$ consists entirely of nilpotent elements. In particular, $C^{\bullet}$ is finite-dimensional, and $H^{\bullet}(\mathcal{L}, \mathbb{F})$ is a graded commutative Cohen-Macaulay $\mathbb{F}$-algebra.

Let $B^{\bullet}$ be a graded commutative $\mathbb{F}$-algebra. Then $B^{\bullet}$ is said to satisfy Poincaré duality in dimension $n$, if $\operatorname{dim}_{\mathbb{F}}\left(B^{n}\right)=1, B^{n+j}=0$ for all $j>0$, and if for all $k \in\{0, \ldots, n\}$ multiplication induces a non-degenerate pairing $B^{k} \otimes B^{n-k} \rightarrow B^{n}$. In [3], Benson and Carlson developed a method for studying the cohomology ring $H^{\bullet}\left(G, \mathbb{F}_{p}\right)$ for a finite group $G$ provided one knows that $H^{\bullet}\left(G, \mathbb{F}_{p}\right)$ is a Cohen-Macaulay $\mathbb{F}$-algebra and $p$ is odd. Their main result can be summarised as follows:

THEOREM 4.4. ([3, Theorem 6.3]) Let $\mathbb{F}$ be a field of characteristic $p \neq 2$, and let $\mathbf{A}$ be a finite-dimensional cocommutative $\mathbb{F}$-Hopf algebra such that

(i) $\mathbf{A}$ is a Frobenius algebra.

(ii) $H^{\bullet}(\mathbf{A}, \mathbb{F})$ is a finitely generated Cohen-Macaulay $\mathbb{F}$-algebra.

Let $\xi_{1}, \ldots, \xi_{n}$ be a homogeneous system of parameters of degree $s_{1}, \ldots, s_{n}, s_{i} \geqslant 2$. Then $C^{\bullet}:=H^{\bullet}(\mathbf{A}, \mathbb{F}) /\left\langle\xi_{1}, \ldots, \xi_{n}\right\rangle$ satisfies Poincaré duality in dimension $s:=\sum_{i=1}^{n}\left(s_{i}-1\right)$.

Proof: The cocommutativity of the Hopf algebra $\mathbf{A}$ ensures that for left $\mathbf{A}$-modules $M$ and $N$, the tensor product $M \otimes_{\mathbb{F}} N$ is a projective left A-module whenever one of the factors is projective. The property of being a Frobenius algebra implies that the left regular A-module $\mathbf{A}$ is also injective (see [1, Proposition 1.6.2]). Therefore one can transfer the proof of $[3$, Theorem 6.3$]$ ad verbatim.

It is well-known that for a finite-dimensional restricted Lie algebra $\mathfrak{L}$, the restricted universal enveloping algebra $u(\mathfrak{L})$ is a Frobenius algebra (see [4]). Moreover, if $p$ is odd and $\mathfrak{L}$ is a $p$-central restricted Lie algebra, Theorem 4.3 has shown that the cohomology ring $H^{\bullet}(\mathfrak{L}, \mathbb{F})$ is a Cohen-Macaulay algebra with a homogeneous system of parameters $\xi_{1}, \ldots, \xi_{n}$ all of degree 2, where $n:=\operatorname{dim}_{F}\left(\mathfrak{L}_{[p]}\right)$. Hence from Theorem 4.4 one obtains:

COROLlary 4.5. The finite-dimensional $\mathbb{F}$-algebra $C^{\bullet}$ of Theorem 4.3 satisfies Poincaré duality in dimension $n:=\operatorname{dim}_{\mathbb{F}}\left(\mathfrak{L}_{[p]}\right)$.

REMARK 4.6. Let $\mathcal{L}$ be a finite-dimensional $p$-central restricted Lie algebra, and let

$$
h_{\mathfrak{L}}(t):=\sum_{k \in \mathbb{N}_{0}} \operatorname{dim}_{\mathbf{F}}\left(H^{k}(\mathfrak{L}, \mathbb{F})\right) \cdot t^{k}
$$

denote the Hilbert series of its cohomology algebra $H^{\bullet}(\mathfrak{L}, \mathbb{F})$. One has a multiplicative decomposition $h_{\mathfrak{L}}(t)=c(t) \cdot\left(1-t^{2}\right)^{-n}$, where $c(t)$ denotes the Hilbert series of $C^{\bullet}$ and 
$n:=\operatorname{dim}_{\mathbf{F}}\left(\mathfrak{L}_{[p]}\right)$. The Poincaré duality of $C^{\bullet}$ implies that $c(t)=t^{n} \cdot c(1 / t)$. Hence $h_{\mathfrak{L}}(t)$ satisfies the functional equation

$$
h_{\mathfrak{L}}(1 / t)=(-t)^{\operatorname{dim}_{\mathbf{F}}\left(\mathcal{L}_{[p \mathfrak{1})}\right)} \cdot h_{\mathfrak{L}}(t)
$$

The analogous functional equation also holds for $p$-central groups. Let $G$ be a finite $p$-central group, that is, $\Omega_{1}(G):=\left\{g \in G \mid g^{p}=1\right\} \leqslant \mathrm{Z}(G)$, and let

$$
h_{G}(t):=\sum_{k \in \mathbb{N}_{0}} \operatorname{dim}_{\mathbb{F}_{p}}\left(H^{k}\left(G, \mathbb{F}_{p}\right)\right) \cdot t^{k}
$$

denote the Hilbert series of the $\bmod p$ cohomology ring of $G$. By [6], $H^{\bullet}\left(G, \mathbb{F}_{p}\right)$ is a Cohen-Macaulay $\mathbb{F}_{p}$-algebra, and thus by [2, Theorem 5.18.1]),

$$
h_{G}(1 / t)=(-t)^{\operatorname{dim}_{p}\left(\Omega_{1}(G)\right)} \cdot h_{G}(t) .
$$

\section{REFERENCES}

[1] D.J. Benson, Representations and cohomology. I, Cambridge Studies in Advanced Mathematics 30 (Cambridge University Press, Cambridge, 1991).

[2] D.J. Benson, Representations and cohomology. II, Cambridge Studies in Advanced Mathematics 31 (Cambridge University Press, Cambridge, 1991).

[3] D.J. Benson and J.F. Carlson, 'Projective resolutions and Poincaré duality complexes', Trans. Amer. Math. Soc. 342 (1994), 447-488.

[4] A.J. Berkson, 'The $u$-algebra of a restricted Lie algebra is Frobenius', Proc. Amer. Math. Soc. 15 (1964), 14-15.

[5] M. Bianchi, A. Gillio Berta Mauri and L. Verardi, 'Groups in which elements with the same p-power permute', Matematiche (Catania) 51 (1996). suppl. (1997) 53-61 .

[6] C. Broto and H-W. Henn, 'Some remarks on central elementary abelian $p$-subgroups and cohomology of classifying spaces', Quart. J. Math. Oxford Ser. (2) 44 (1993), 155-163.

[7] J. Buckley, 'Finite groups whose minimal subgroups are normal', Math. Z. 116 (1970), 15-17.

[8] X.K. Du, 'The centers of a radical ring', Canad. Math. Bull. 35 (1992), 174-179.

[9] J. González-Sánchez and A. Jaikin-Zapirain, 'On the structure of normal subgroups of potent p-groups', J. Algebra 276 (2004), 193-209.

[10] G. Hochschild, 'Cohomology of restricted Lie algebras', Amer. J. Math. 76 (1954), $555-580$.

[11] J.C. Jantzen, 'Kohomologie von $p$-Lie-Algebren und nilpotente Elemente', Abh. Math. Sem. Univ. Hamburg 56 (1986), 191-219.

[12] J.C. Jantzen, 'Restricted Lie algebra cohomology', in Algebraic groups Utrecht 1986, Lecture Notes in Math. 1271 (Springer-Verlag, Berlin, 1987), pp. 91-108.

[13] A.V. Kelarev, 'Directed graphs and Lie superalgebras of matrices', J. Algebra 285 (2005), $1-10$.

[14] A.V. Kelarev, Ring constructions and applications, Series in Algebra 9 (World Scientific Publishing Co. Inc., River Edge, NJ, 2002). 
[15] A. Lubotzky and A. Mann, 'Powerful p-groups. I. Finite groups', J. Algebra 105 (1987), 484-505.

[16] A. Lubotzky and A. Mann, 'Powerful p-groups. II. p-adic analytic groups', J. Algebra $105,506-515$.

[17] J.W. Milnor and J.C. Moore, 'On the structure of Hopf algebras', Ann. of Math. (2) 81 (1965), 211-264.

[18] D. Quillen, 'The spectrum of an equivariant cohomology ring. I, II', Ann. of Math. (2) 94 (1971), 549-572. ibid. (2) 94 (1971), 573-602.

[19] D.M. Riley and J.F. Semple, 'Completion of restricted Lie algebras', Israel J. Math. 86 (1994), 277-299.

[20] D.M. Riley and A. Shalev, 'The Lie structure of enveloping algebras', J. Algebra 162 (1993), 46-61.

[21] D.M. Riley and A. Shalev, 'Restricted Lie algebras and their envelopes', Canad. J. Math. 47 (1995), 146-164.

[22] D.M. Riley and V. Tasić, 'Lie identities for Hopf algebras', J. Pure Appl. Algebra 122 (1997), 127-134.

[23] S. Siciliano, 'Lie derived lengths of restricted universal enveloping algebras', Publ. Math. Debrecen 68 (2006), 503-513.

[24] S. Siciliano and E. Spinelli, 'Lie nilpotency indices of restricted universal enveloping algebras', Comm. Algebra 34 (2006), 151-157.

[25] S. Siciliano and E. Spinelli, 'Lie metabelian restricted universal enveloping algebras', Arch. Math. (Basel) 84 (2005), 398-405.

[26] H. Strade and R. Farnsteiner, Modular Lie algebras and their representations, Monographs and Textbooks in Pure and Applied Mathematics 116 (Marcel Dekker Inc., New York, 1988).

[27] P. Symonds and Th. Weigel, 'Cohomology of p-adic analytic groups', in New horizons in pro-p groups, (M. duSautoy, D. Segal and A. Shalev, Editors), Progress in Mathematics 184 (Birkhäuser, Boston, 2000), pp. 349-410.

[28] Th. Weigel, 'p-central groups and Poincaré duality', Trans. Amer. Math. Soc. 352 (2000), 4143-4154.

Dipartimento di Matematica "E. De Giorgi"

Universitá di Lecce

Via prov. Lecce-Arnesano

73100 Lecce

Italy

e-mail: salvatore.siciliano@unile.it
Universitá di Milano-Bicocca

U5-3067, Via R.Cozzi, 53

20125 Milano

Italy

e-mail: thomas.weigel@unimib.it 